\title{
Knowledge of Pregnant Women about Pregnancy Gingivitis and Children Oral Health
}

\author{
Rafi A. Togoo ${ }^{1}$ Balsam Al-Almai ${ }^{1}$ Fatimah Al-Hamdi ${ }^{1}$ \\ ${ }^{1}$ Department of Pediatric Dentistry and Orthodontics King Khalid \\ University College of Dentistry Abha, Kingdom of Saudi Arabia
}

\author{
Salha H. Huaylah $\quad$ Mashael Althobati ${ }^{1} \quad$ Safeyah Alqarni ${ }^{1}$
}

\begin{abstract}
Address for correspondence Salha H. Huaylah, BDS, Department of Pediatric Dentistry and Orthodontics King Khalid University College of Dentistry Abha, Kingdom of Saudi Arabia (e-mail: salhahuaylah@gmail.com).
\end{abstract}

\begin{abstract}
Keywords

- pregnancy

- gingivitis

- knowledge

- women

Objective A cross-sectional questionnaire-based study was carried out among 251 pregnant women to assess their level of knowledge and perception regarding pregnancy gingivitis and the oral health of new born children.

Materials and Methods A self-administered validated questionnaire was distributed among pregnant women visiting the Child and Maternity Hospital, Ministry of Health, Aseer, Saudi Arabia. The questionnaire included the questions related to their knowledge/awareness about cause, treatment, preventive measures, and the adverse effects of gingivitis on oral health of a woman.

Statistical Analysis The data was entered into Microsoft excel sheet and put to statistical analysis using SPSS (version 17). Descriptive analysis and also chi-squared test was used for comparison.

Results Majority of pregnant women were found unaware about developing of pregnancy gingivitis as well as the cause, effects, treatment, and preventive measures for the same. Most of the subjects agreed that it was essentially required to have oral health education before their pregnancy term would start.

Conclusion Most of the pregnant women said that they were unaware about pregnancy gingivitis, though few reported of being aware about it. It is inferred that the collaborative efforts between general dentists, pediatric dentists, public health dentists, and medical professionals are needed to raise awareness among pregnant women regarding timely dental care to avoid the adverse pregnancy related outcomes due to gingivitis and periodontitis.
\end{abstract}

\section{Introduction}

Various physiological changes take place through the body of a pregnant woman that include both general and those related to oral cavity. ${ }^{1}$ Due to hormonal fluctuations during pregnancy, alterations occur in the levels of estrogen and progesterone resulting in tortuous and dilated microvasculature, more permeability of oral blood vessels, and lowering of host immunity, which eventually cause the host to become more vulnerable to oral infections. ${ }^{2,3}$ Both reversible and irreversible changes take place in oral cavities of women during pregnancy. High levels of estrogen have been found to be associated with occurrence of gingival hyperplasia, gingivitis, pyogenic granulomas, dental caries, and alterations in salivary flow. ${ }^{4}$ In the studies conducted previously, oral health of pregnant women, adverse pregnancy outcomes (such as preterm births, low birth weight), and oral health of infants have all been linked to each other. ${ }^{5}$

Pregnancy leads not only to the physiological changes in a woman but it also has a menacing impact on her behavioral as well as dietary habits. With the increased need of energy, a pregnant woman has to modify the quantity and content of 
diet that ultimately increases the carbohydrate quantum of food, resulting in more cariogenic food. al-Kanhal and Bani, in their study on pregnant women in Saudi Arabia, reported that majority of women desired for more and more sweets, dates, milk, and salty and sour food, hence increasing their risk of dental caries and dental erosion. ${ }^{6}$

Oral health promotion and relevant oral health education, which would acquaint them regarding timely treatment and preventive measures for gaining optimal oral health, are imperative to be imparted to women especially during the child-bearing age. Women, during their pregnancy, face a number of problems in utilizing the available oral health services due to which their oral health gets deteriorated. They lack knowledge about the appropriate timing for getting their dental treatment done during or before pregnancy. Some of them suffer from negative attitude toward seeking dental care that might be related to their past experience and/or misconception about it. At times, even the dentists show reluctance to treat pregnant women that adds to the negligence of pregnant women toward oral health. ${ }^{7}$

Hence, a study was conducted in Aseer, Saudi Arabia among pregnant women to assess their knowledge and perception toward pregnancy gingivitis and oral health of their children.

\section{Materials and Methods}

A cross-sectional questionnaire-based study was carried out among 18 to 40 years old pregnant women, who visited the Child and Maternity Hospital, Aseer, Saudi Arabia. Prior to conducting of study, ethical approval was obtained from Scientific Research Committee, Aseer, Saudi Arabia. A total convenient sample of 251 pregnant women was included in the study. All the pregnant women who were permanent residents of Saudi Arabia, belonged to age group of 18 to 40 years and gave the consent of participation in the research, were included in the study, while all those who suffered from any systemic ailments such as diabetes and bleeding disorders were excluded from the study.

A close-ended questionnaire was formulated and its reliability was checked by conducting a pilot study on 20 pregnant women who were not a part of the final study sample. The questions were checked for ease in understanding and having clarity by repeating the study on the same respondents. Their responses were thereby checked for reliability using Kappa statistics that yielded satisfactory results $(0.81)$.

The final questionnaire (Appendix A) included two sections: in the first section demographic data such as age and educational qualification of the participants were recorded and in the second section questions related to awareness and knowledge about oral health of women were enquired; as for instance those related to pregnancy gingivitis, its effect on oral health of women, its impact on their new born children, the preventive and treatment measures related to pregnancy gingivitis, and their frequency of visiting the dentist during pregnancy. Moreover, the questionnaire pertained to subject's knowledge regarding the effect of poor oral health on pregnancy outcomes such as preterm births and low birth weights.
An 11-item questionnaire, in both English and Arabic languages, was used and the subjects were interviewed face-to-face so that any difficulty that might creep up in understanding the questions could be explained to the subjects by interviewer. Confidentiality of the responses and identity of the patients were scrupulously maintained and assured so as to avoid nonresponse and social desirability bias.

The data was entered in Microsoft Excel sheet and put to statistical analysis using SPSS (version 17). Descriptive analysis and chi-squared tests were used for comparison.

\section{Results}

In the present study, the age of pregnant women, who participated, ranged between 27 and 39 years, with mean age of 31.80 years ( $\mathrm{df}=3.36$ ). Majority $(42.62 \%$ ) of the pregnant women had just heard about developing pregnancy gingivitis and they were not fully aware about it and $31.47 \%$ had not even heard about it, whereas $25.89 \%$ were aware about it. Majority of women (41.03\%) did not get their dental checkup done during pregnancy even after many of them (28.68\%) had developed reddened and swollen gums. The cause of pregnancy gingivitis was not known to $32.66 \%$ of the pregnant women. As many as $35.05 \%$ respondents felt that daily tooth brushing and flossing would prevent pregnancy gingivitis (-Table 1). Yet as many as $28.68 \%$ pregnant women thought that dentists would prefer medications as a treatment of pregnancy gingivitis, while $19.92 \%$ did not know about it. Around $27 \%$ of subjects considered tooth sensitivity to be the main consequence of pregnancy gingivitis. When enquired whether pregnancy gingivitis could affect the oral health of a new born, a majority of women (47.41\%) were undecided about it, while $28.68 \%$ were unaware of any such effect on the oral health of the new born. Most of the pregnant women $(55.37 \%)$ were unaware about the adverse pregnancy outcome, associated with pregnancy gingivitis (-Table 2 ). When the responses of pregnant women were compared, based on the level of education, a statistically significant difference was found between the responses of all the questions ( $p \leq 0.05$ ). Respondents to the tune of $49 \%$ reported that they used moist towels for cleaning oral cavity of their children who were less than 18 months of age (-Fig. 1). Majority of subjects believed positively to receive dental education compulsorily at the start of their pregnancy term ( - Fig. 2).

\section{Discussion}

Inflammation of soft tissues surrounding the teeth, without loss of attachment, is known as gingivitis, while periodontitis involves damage of supporting tooth structures. The inflammation of gums that is exaggerated during the period of pregnancy is known as pregnancy gingivitis given rise due to changes in hormonal levels and in that of life style of a pregnant woman. In various previous studies, poor oral health such as periodontitis in a pregnant woman has been associated with various adverse pregnancy outcomes such as preterm births and low birth weights. ${ }^{8}$ Increase in the hormonal levels of estrogen and progesterone can cause 
Table 1 Frequency distribution of responses according to education level of subjects

\begin{tabular}{|l|l|l|l|l|l|l|l|}
\hline $\begin{array}{l}\text { Q1) Being aware } \\
\text { about developing } \\
\text { pregnancy } \\
\text { gingivitis during } \\
\text { pregnancy }\end{array}$ & $\begin{array}{l}\text { Total no. of } \\
\text { respondents } \\
\text { (\%age) }\end{array}$ & $\begin{array}{l}\text { Illiterate } \\
\text { women }\end{array}$ & $\begin{array}{l}\text { Primary } \\
\text { school }\end{array}$ & $\begin{array}{l}\text { High } \\
\text { school }\end{array}$ & $\begin{array}{l}\text { Higher } \\
\text { secondary } \\
\text { school }\end{array}$ & Graduate & Postgraduate \\
\hline Yes, I am fully aware & $65(25.89 \%)$ & $1(1.53 \%)$ & $2(3.07 \%)$ & $3(4.61 \%)$ & $6(9.2 \%)$ & $8(12.30 \%)$ & $45(69.23 \%)$ \\
\hline No, I'm not aware & $\begin{array}{l}79 \\
(31.47 \%)\end{array}$ & $\begin{array}{l}22 \\
(27.84 \%)\end{array}$ & $\begin{array}{l}9 \\
(11.39 \%)\end{array}$ & $\begin{array}{l}20 \\
(25.31 \%)\end{array}$ & $12(15.18 \%)$ & $14(17.72 \%)$ & $2(2.53 \%)$ \\
\hline $\begin{array}{l}\text { Not fully aware, but } \\
\text { heard it }\end{array}$ & $\begin{array}{l}107 \\
(\mathbf{4 2 . 6 2 \% )}\end{array}$ & $\begin{array}{l}22 \\
(20.56 \%)\end{array}$ & $\begin{array}{l}17 \\
(15.88 \%)\end{array}$ & $\begin{array}{l}26 \\
(24.29 \%)\end{array}$ & $21(19.62 \%)$ & $20(18.69 \%)$ & $1(0.93 \%)$ \\
\hline
\end{tabular}

Chi-squared test $=149.54, \mathrm{df}=12, p$-Value $=0.0000$

\begin{tabular}{|l|l|l|l|l|l|l|l|}
\hline $\begin{array}{l}\text { Q2) Getting the } \\
\text { dental checkup } \\
\text { done during or } \\
\text { before pregnancy }\end{array}$ & $\begin{array}{l}\text { Total no. of } \\
\text { respondents } \\
\text { (\%age) }\end{array}$ & $\begin{array}{l}\text { Illiterate } \\
\text { women }\end{array}$ & $\begin{array}{l}\text { Primary } \\
\text { school }\end{array}$ & $\begin{array}{l}\text { High } \\
\text { school }\end{array}$ & $\begin{array}{l}\text { Higher } \\
\text { secondary } \\
\text { school }\end{array}$ & Graduate & Postgraduate \\
\hline No, I didn't & $\begin{array}{l}103 \\
(\mathbf{4 1 . 0 3 \% )}\end{array}$ & $\begin{array}{l}35 \\
(33.98 \%)\end{array}$ & $\begin{array}{l}18 \\
(17.47 \%)\end{array}$ & $\begin{array}{l}21 \\
(20.38 \%)\end{array}$ & $21(20.38 \%)$ & $6(5.82 \%)$ & $8(7.76 \%)$ \\
\hline Yes, I did & $62(24.8 \%)$ & $3(4.83 \%)$ & $4(6.45 \%)$ & $\begin{array}{l}11 \\
(17.74 \%)\end{array}$ & $11(17.74 \%)$ & $22(35.48 \%)$ & $24(38.70 \%)$ \\
\hline $\begin{array}{l}\text { Yes I planned to but } \\
\text { did not go. }\end{array}$ & $49(19.52)$ & $\begin{array}{l}6 \\
(12.24 \%)\end{array}$ & $2(4.08 \%)$ & $\begin{array}{l}8 \\
(16.32 \%)\end{array}$ & $8(16.32 \%)$ & $8(16.32 \%)$ & $6(12.24 \%)$ \\
\hline $\begin{array}{l}\text { Yes, due to dental } \\
\text { condition }\end{array}$ & $37(14.74 \%)$ & $1(2.70 \%)$ & $\begin{array}{l}4 \\
(10.81 \%)\end{array}$ & $\begin{array}{l}9 \\
(24.32 \%)\end{array}$ & $6(24.32 \%)$ & $6(16.21 \%)$ & $10(27.02 \%)$ \\
\hline
\end{tabular}

\begin{tabular}{|c|c|c|c|c|c|c|c|}
\hline \multicolumn{8}{|c|}{ Chi-squared test $=73.867, \mathrm{df}=15, p$-Value $=0.0000$} \\
\hline $\begin{array}{l}\text { Q3) Do you have } \\
\text { any following } \\
\text { signs of pregnancy } \\
\text { gingivitis }\end{array}$ & $\begin{array}{l}\text { Total no. of } \\
\text { respondents } \\
\text { (\%age) }\end{array}$ & $\begin{array}{l}\text { Illiterate } \\
\text { women }\end{array}$ & $\begin{array}{l}\text { Primary } \\
\text { school }\end{array}$ & $\begin{array}{l}\text { High } \\
\text { school }\end{array}$ & $\begin{array}{l}\text { Higher } \\
\text { secondary } \\
\text { school }\end{array}$ & Graduate & Postgraduate \\
\hline $\begin{array}{l}\text { Swollen \& reddened } \\
\text { gums }\end{array}$ & $72(28.68 \%)$ & $\begin{array}{l}14 \\
(19.44 \%)\end{array}$ & $6(8.33 \%)$ & $\begin{array}{l}14 \\
(19.44 \%)\end{array}$ & $9(12.5 \%)$ & $12(16.66 \%)$ & $14(19.44 \%)$ \\
\hline Bad breath & $65(25.89 \%)$ & $\begin{array}{l}12 \\
(18.46 \%)\end{array}$ & $\begin{array}{l}8 \\
(12.30 \%)\end{array}$ & $\begin{array}{l}11 \\
(16.92 \%)\end{array}$ & $8(12.30 \%)$ & $10(15.38 \%)$ & $10(15.38 \%)$ \\
\hline Gum bleeding & $69(27.49 \%)$ & $\begin{array}{l}14 \\
(20.28 \%)\end{array}$ & $\begin{array}{l}11 \\
(15.94 \%)\end{array}$ & $6(8.69 \%)$ & $7(10.14 \%)$ & $8(11.59 \%)$ & $12(17.39 \%)$ \\
\hline $\begin{array}{l}\text { Red spot over oral } \\
\text { lining }\end{array}$ & $10(3.98)$ & $4(40 \%)$ & $2(20 \%)$ & $5(50 \%)$ & $2(20 \%)$ & $0(0 \%)$ & $0(0 \%)$ \\
\hline None & 35 (13.94\%) & $1(2.85 \%)$ & $1(2.85 \%)$ & $3(8.57 \%)$ & $13(37.14 \%)$ & $12(34.28 \%)$ & $12(34.28 \%)$ \\
\hline
\end{tabular}

Chi-squared test $=38.631, \mathrm{df}=\mathbf{2 0}, \mathrm{p}$-Value $=\mathbf{0 . 0 0 7 1}$

\begin{tabular}{|c|c|c|c|c|c|c|c|}
\hline $\begin{array}{l}\text { Q4) What may } \\
\text { be the cause of } \\
\text { gingivitis during } \\
\text { pregnancy }\end{array}$ & $\begin{array}{l}\text { Total no. of } \\
\text { respondents } \\
\text { (\%age) }\end{array}$ & $\begin{array}{l}\text { Illiterate } \\
\text { women }\end{array}$ & $\begin{array}{l}\text { Primary } \\
\text { school }\end{array}$ & $\begin{array}{l}\text { High } \\
\text { school }\end{array}$ & $\begin{array}{l}\text { Higher } \\
\text { secondary } \\
\text { school }\end{array}$ & Graduate & Postgraduate \\
\hline Hormonal changes & 78 (31.07\%) & $2(2.56 \%)$ & $2(2.56 \%)$ & $\begin{array}{l}14 \\
(17.94 \%)\end{array}$ & $12(15.38 \%)$ & 20 (25.64\%) & $22(28.20 \%)$ \\
\hline $\begin{array}{l}\text { Traumatic tooth } \\
\text { brushing }\end{array}$ & $20(7.96)$ & $9(45 \%)$ & $2(10 \%)$ & $4(20 \%)$ & $2(10 \%)$ & $1(5 \%)$ & $2(10 \%)$ \\
\hline Poor oral hygiene & 45 (17.92\%) & $2(4.44 \%)$ & $3(6.66 \%)$ & $9(20 \%)$ & $9(20 \%)$ & $14(31.11 \%)$ & $11(24.44 \%)$ \\
\hline Eating fried food & $6(2.39 \%)$ & $\begin{array}{l}2 \\
(33.33 \%)\end{array}$ & $\begin{array}{l}1 \\
(16.66 \%)\end{array}$ & $\begin{array}{l}2 \\
(33.33 \%)\end{array}$ & $2(33.33 \%)$ & $1(16.66 \%)$ & $3(50 \%)$ \\
\hline Malnutrition & $20(7.96 \%)$ & $8(40 \%)$ & $8(40 \%)$ & $2(10 \%)$ & $1(5 \%)$ & $0(0 \%)$ & $3(15 \%)$ \\
\hline Do not know & $82(32.66 \%)$ & $\begin{array}{l}22 \\
(26.82 \%)\end{array}$ & $\begin{array}{l}12 \\
(14.63 \%)\end{array}$ & $\begin{array}{l}18 \\
(21.95 \%)\end{array}$ & $13(15.85 \%)$ & $6(7.31 \%)$ & $7(8.53 \%)$ \\
\hline
\end{tabular}


continued

\begin{tabular}{|c|c|c|c|c|c|c|c|}
\hline $\begin{array}{l}\text { Q5) Measures } \\
\text { that you think can } \\
\text { prevent Pregnancy } \\
\text { gingivitis }\end{array}$ & $\begin{array}{l}\text { Total no. of } \\
\text { respondents } \\
\text { (\%age) }\end{array}$ & $\begin{array}{l}\text { Illiterate } \\
\text { women }\end{array}$ & $\begin{array}{l}\text { Primary } \\
\text { school }\end{array}$ & $\begin{array}{l}\text { High } \\
\text { schooling }\end{array}$ & $\begin{array}{l}\text { Higher } \\
\text { secondary } \\
\text { school }\end{array}$ & Graduate & Postgraduate \\
\hline $\begin{array}{l}\text { Daily tooth brushing } \\
\text { and flossing }\end{array}$ & $88(35.05 \%)$ & $\begin{array}{l}15 \\
(17.04 \%)\end{array}$ & $\begin{array}{l}11 \\
(12.5 \%)\end{array}$ & $\begin{array}{l}17 \\
(19.31 \%)\end{array}$ & $11(12.5 \%)$ & $14(15.90 \%)$ & $18(20.45 \%)$ \\
\hline Balanced diet & 55 (21.91\%) & $\begin{array}{l}12 \\
(21.81 \%)\end{array}$ & $3(5.45 \%)$ & $11(20 \%)$ & $12(21.81 \%)$ & $9(16.36 \%)$ & $10(18.18 \%)$ \\
\hline $\begin{array}{l}\text { Dental checkup } \\
\text { before pregnancy }\end{array}$ & $58(23.10 \%)$ & $\begin{array}{l}9 \\
(15.51 \%)\end{array}$ & $\begin{array}{l}10 \\
(17.24 \%)\end{array}$ & $\begin{array}{l}13 \\
(22.41 \%)\end{array}$ & $7(12.06 \%)$ & $9(15.51 \%)$ & $13(22.41 \%)$ \\
\hline Monthly Scaling & $10(3.98 \%)$ & $3(30 \%)$ & $0(0 \%)$ & $2(20 \%)$ & $2(20 \%)$ & $0(0 \%)$ & $3(30 \%)$ \\
\hline $\begin{array}{l}\text { Dental checkup } \\
\text { during second } \\
\text { trimester }\end{array}$ & $32(12.74 \%)$ & $2(6.25 \%)$ & $1(3.12 \%)$ & $\begin{array}{l}6 \\
(18.75 \%)\end{array}$ & $7(21.87 \%)$ & 10 (31.25\%) & $4(12.5 \%)$ \\
\hline Do not know & $8(3.18 \%)$ & $4(50 \%)$ & $3(37.5 \%)$ & $0(0 \%)$ & $0(0 \%)$ & $0(0 \%)$ & $0(0 \%)$ \\
\hline
\end{tabular}

Table 2 Frequency distribution of responses according to education level of subjects

\begin{tabular}{|c|c|c|c|c|c|c|c|}
\hline $\begin{array}{l}\text { Q6) What as per } \\
\text { your knowledge, } \\
\text { is the treatment } \\
\text { dentists provide for } \\
\text { pregnancy gingivitis }\end{array}$ & $\begin{array}{l}\text { Total no. of } \\
\text { respondents }\end{array}$ & Illiterate & $\begin{array}{l}\text { Primary } \\
\text { school }\end{array}$ & $\begin{array}{l}\text { High } \\
\text { school }\end{array}$ & $\begin{array}{l}\text { Higher } \\
\text { secondary } \\
\text { school }\end{array}$ & Graduate & Postgraduate \\
\hline $\begin{array}{l}\text { Surgical removal of } \\
\text { swollen gum }\end{array}$ & $10(3.98 \%)$ & $5(50 \%)$ & $1(10 \%)$ & $2(20 \%)$ & $1(10 \%)$ & $1(10 \%)$ & $2(20 \%)$ \\
\hline $\begin{array}{l}\text { No treatment } \\
\text { needed }\end{array}$ & 35 (13.94\%) & $8(22.85 \%)$ & $5(14.28 \%)$ & 9 (25.71\%) & $4(11.42 \%)$ & $1(2.85 \%)$ & $2(5.71 \%)$ \\
\hline Professional scaling & 60 (23.90\%) & $1(1.66 \%)$ & $1(1.66 \%)$ & $5(8.33 \%)$ & $15(25 \%)$ & $24(40 \%)$ & $28(46.66 \%)$ \\
\hline $\begin{array}{l}\text { Extract the affected } \\
\text { tooth }\end{array}$ & 24 (9.56\%) & $7(29.16 \%)$ & $7(29.16 \%)$ & $2(8.33 \%)$ & $1(4.16 \%)$ & $1(4.16 \%)$ & $0(0 \%)$ \\
\hline Medications & 72 (28.68\%) & $8(11.11 \%)$ & $5(6.94 \%)$ & $\begin{array}{l}17 \\
(23.61 \%)\end{array}$ & 15 (20.83\%) & $13(18.05 \%)$ & 15 (20.83\%) \\
\hline Do not know & 50 (19.92\%) & $16(32 \%)$ & $9(18 \%)$ & $14(28 \%)$ & $3(6 \%)$ & $2(4 \%)$ & $1(2 \%)$ \\
\hline \multicolumn{8}{|c|}{ Chi-squared test $=119.645, \mathrm{df}=25, p$-Value $=0.0000$} \\
\hline $\begin{array}{l}\text { Q7) What affect can } \\
\text { pregnancy gingivitis } \\
\text { have on your oral } \\
\text { health? }\end{array}$ & $\begin{array}{l}\text { Total no. of } \\
\text { respondents }\end{array}$ & Illiterate & $\begin{array}{l}\text { Primary } \\
\text { school }\end{array}$ & $\begin{array}{l}\text { High } \\
\text { school }\end{array}$ & $\begin{array}{l}\text { Higher } \\
\text { secondary } \\
\text { school }\end{array}$ & Graduate & Postgraduate \\
\hline $\begin{array}{l}\text { Overgrowth of gum } \\
\text { tissue }\end{array}$ & 27 (10.75\%) & $2(7.40 \%)$ & $1(3.70 \%)$ & $3(11.11 \%)$ & $5(18.51 \%)$ & $6(22.22 \%)$ & 7 (25.92\%) \\
\hline No effect & $22(8.76 \%)$ & $\begin{array}{l}13 \\
(59.09 \%)\end{array}$ & $4(18.18 \%)$ & $3(13.63 \%)$ & 0 & $1(4.54 \%)$ & $1(4.54 \%)$ \\
\hline Periodontal disease & $34(13.54 \%)$ & $2(5.88 \%)$ & $3(8.82 \%)$ & $8(23.52 \%)$ & $6(17.64 \%)$ & 7 (20.58\%) & $8(23.52 \%)$ \\
\hline Tooth decay & $40(15.93 \%)$ & $11(27.5 \%)$ & $7(17.5 \%)$ & $12(30 \%)$ & $7(17.5 \%)$ & $2(5 \%)$ & $1(2.5 \%)$ \\
\hline Tooth sensitivity & 67 (26.69\%) & $4(5.97 \%)$ & $3(4.47 \%)$ & $\begin{array}{l}11 \\
(16.41 \%) \\
\end{array}$ & $\begin{array}{l}13 \\
(19.40 \%)\end{array}$ & $\begin{array}{l}14 \\
(20.89 \%)\end{array}$ & $21(31.34 \%)$ \\
\hline Loose tooth & $30(11.95 \%)$ & $1(3.33 \%)$ & $1(3.33 \%)$ & $4(13.33 \%)$ & $5(16.66 \%)$ & $11(36.66 \%)$ & $9(30 \%)$ \\
\hline Do not know & $31(12.35 \%)$ & $\begin{array}{l}12 \\
(38.70 \%)\end{array}$ & $9(29.03 \%)$ & $8(25.80 \%)$ & $3(9.67 \%)$ & $1(3.22 \%)$ & $1(3.22 \%)$ \\
\hline
\end{tabular}


continued

\begin{tabular}{|c|c|c|c|c|c|c|c|}
\hline $\begin{array}{l}\text { Q8). Will pregnancy } \\
\text { gingivitis affect the } \\
\text { oral health of new } \\
\text { born? }\end{array}$ & $\begin{array}{l}\text { Total no. of } \\
\text { respondents }\end{array}$ & Illiterate & $\begin{array}{l}\text { Primary } \\
\text { school }\end{array}$ & $\begin{array}{l}\text { High } \\
\text { school }\end{array}$ & $\begin{array}{l}\text { Higher } \\
\text { secondary } \\
\text { school }\end{array}$ & Graduate & Postgraduate \\
\hline Yes, it will & $60(23.90 \%)$ & $3(5 \%)$ & $2(3.33 \%)$ & $12(20 \%)$ & $11(18.33 \%)$ & $15(25 \%)$ & $24(40 \%)$ \\
\hline No, it won't & $72(28.68 \%)$ & $18(25 \%)$ & $\begin{array}{l}12 \\
(16.66 \%)\end{array}$ & $\begin{array}{l}16 \\
(22.22 \%)\end{array}$ & $4(5.55 \%)$ & $8(11.11 \%)$ & $8(11.11 \%)$ \\
\hline Undecided & $\begin{array}{l}119 \\
(47.41 \%)\end{array}$ & $\begin{array}{l}24 \\
(20.16 \%)\end{array}$ & $\begin{array}{l}14 \\
(11.76 \%)\end{array}$ & $\begin{array}{l}21 \\
(17.64 \%)\end{array}$ & $24(20.16 \%)$ & $19(15.96 \%)$ & $16(13.44 \%)$ \\
\hline \multicolumn{8}{|c|}{ Chi-squared test $=39.4812, \mathrm{df}=10, p$-Value $=0.00002$} \\
\hline $\begin{array}{l}\text { Q9) What can } \\
\text { be the adverse } \\
\text { pregnancy outcome } \\
\text { of pregnancy } \\
\text { gingivitis? }\end{array}$ & $\begin{array}{l}\text { Total no. of } \\
\text { respondents }\end{array}$ & Illiterate & $\begin{array}{l}\text { Primary } \\
\text { school }\end{array}$ & $\begin{array}{l}\text { High } \\
\text { school }\end{array}$ & $\begin{array}{l}\text { Higher } \\
\text { secondary } \\
\text { school }\end{array}$ & Graduate & Postgraduate \\
\hline Preterm birth & 34 (13.54\%) & $1(2.94 \%)$ & 1 (2.94\%) & $2(5.88 \%)$ & $10(29.41 \%)$ & $10(29.41 \%)$ & 22 (64.70\%) \\
\hline No effect & $55(21.91)$ & $\begin{array}{l}15 \\
(27.27 \%)\end{array}$ & $8(14.54 \%)$ & $\begin{array}{l}13 \\
(23.63 \%)\end{array}$ & $7(12.72 \%)$ & $7(12.72 \%)$ & $5(9.09 \%)$ \\
\hline $\begin{array}{l}\text { Malformation of } \\
\text { bones }\end{array}$ & $7(2.78 \%)$ & $1(14.28 \%)$ & $1(14.28 \%)$ & 1 (14.28\%) & $4(57.14 \%)$ & $4(57.14 \%)$ & $1(14.28 \%)$ \\
\hline Cleft Lip/Cleft palate & $16(6.37 \%)$ & $0(0 \%)$ & $1(6.25 \%)$ & 1 (6.25\%) & $4(25 \%)$ & $4(25 \%)$ & 7 (43.75\%) \\
\hline Do not Know & $\begin{array}{l}139 \\
(55.37 \%)\end{array}$ & $\begin{array}{l}28 \\
(20.14 \%)\end{array}$ & $\begin{array}{l}17 \\
(12.23 \%)\end{array}$ & $\begin{array}{l}32 \\
(23.02 \%)\end{array}$ & 17 (12.23\%) & $17(12.23 \%)$ & 13 (9.39\%) \\
\hline
\end{tabular}

hyperaemia, edema, and bleeding in periodontal tissues; and these are the risk factors for bacterial infections. ${ }^{9}$ Hence, there arise a need to know the attitude of pregnant women toward their dental care so that oral health promotion strategies can be aimed at in the required direction for early diagnosis and treatment of pregnancy gingivitis and periodontitis, hence leading to prevention of its probable adverse outcomes on new born babies. Thus, the present study was conducted among the child-bearing age group of pregnant women to assess their knowledge, attitude, and perception of pregnancy gingivitis.

When enquired about their awareness of developing gingivitis during pregnancy, majority (42.62\%) of the pregnant women said that they had only heard about developing pregnancy gingivitis and were not fully aware about it; other $31.47 \%$ of the respondents had not even heard about it, while $25.89 \%$ of them were aware about the same. Majority of the women who were fully aware about pregnancy gingivitis were graduates/postgraduates in sync with their educational level, depicting a statistically significant difference between the responses of educated and uneducated respondents. These findings indicate that education has a diverse set of impact on the oral health awareness during pregnancy as is mentioned in previous study conducted by Sunita Bamanikar and Liew KokKee wherein they found vivid difference in good oral health knowledge in educated pregnant women in comparison of those having a little or no education; the reason thereof might be their better social communication with others and hence sharing of views and ideas leading to increase in their level of oral health-related knowledge. ${ }^{10}$
In the present study, when the participants were enquired about getting their dental checkup done during their course of pregnancy, majority of them (41.03\%) said they did not get their dental checkup done during or before pregnancy. This finding is similar with the previous studies in which it was reported that women decrease or stop visiting the dentists for dental checkups during pregnancy. ${ }^{11,12}$

This finding is a pointer to misconceptions which the women are having regarding dental care especially during or before pregnancy. It is in contrast to the fact that dental checkup should be undertaken by pregnant women before their pregnancy so that the dentists are able to evaluate their oral health conditions and educate their patients about the expected changes in their oral tissues especially gingiva and also about the preventive measures to be adopted and practiced so as to avoid oral health-related problems. The misconception of harm to the fetus due to dental checkup or dental-treatment leads to aversion of pregnant women to a dentist that ultimately endangers them as well as the oral health of child at later stages. ${ }^{13}$

A good number of respondents (28.68\%) agreed that they had swollen and reddened gums; $27.49 \%$ of them complained of having bleeding gums, yet other $25.89 \%$ said of having bad breath, whereas only $13.9 \%$ felt that they had no signs of pregnancy gingivitis. These findings are in concord with previous studies, wherein they found the symptoms like oral malador, bleeding gums, dental caries, dry mouth, gingival redness persisting among pregnant women; and also stress being one of the causal factors for such poor oral health. ${ }^{14,15}$ 


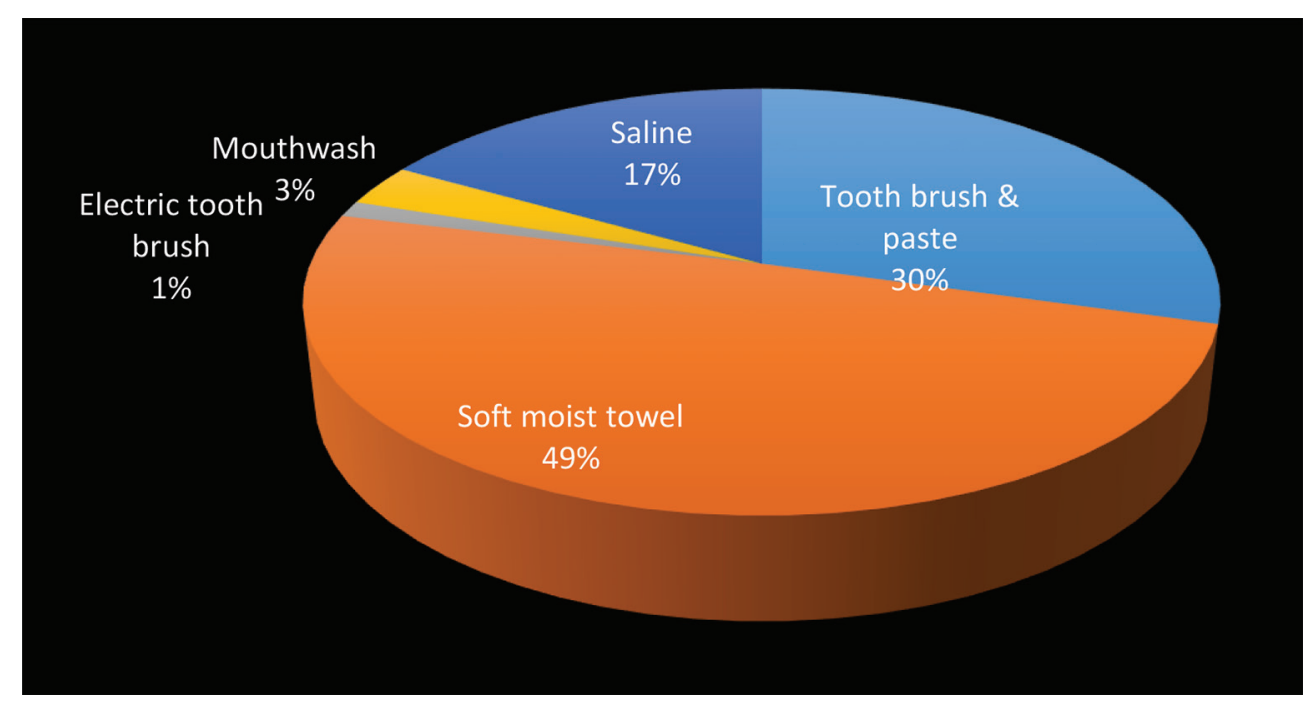

Fig. 1 Frequency distribution of respondents regarding cleaning of oral cavity of children less than 18 months old.

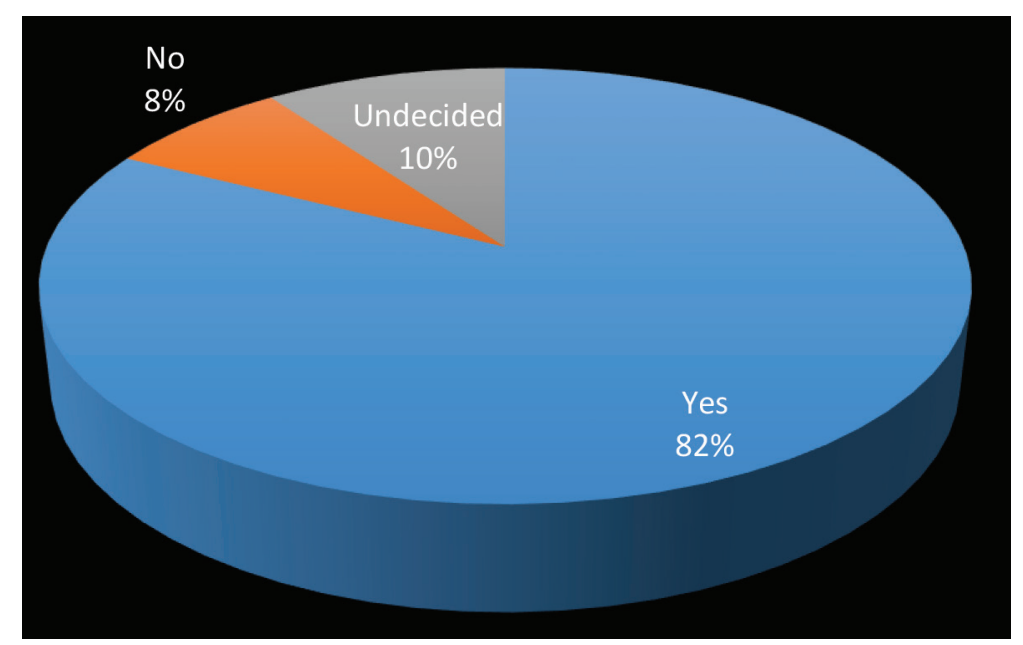

Fig. 2 Frequency distribution of respondents regarding their view about compulsorily having dental education at the start of pregnancy term.

In the present study, $32.66 \%$ of respondents did not know about the cause of pregnancy gingivitis, while $31.07 \%$ of them reported the hormonal changes to be the cause for the same and $17.92 \%$ considered poor oral hygiene as the cause for pregnancy gingivitis. Hormonal changes during pregnancy have been reported in various previously conducted studies as being the main cause of oral tissue changes inducing pregnancy gingivitis, periodontitis, and dental caries. During pregnancy, there is an increase in the metabolism of estrogen by gingiva and also prostaglandins that are synthesized, cause alterations in gingival tissues. ${ }^{16}$ The immune system decreases due to change in hormonal (estrogen and progesterone) levels, which has a bearing on the rate of collagen production; and hence body becomes unable to repair the gingiva. ${ }^{17}$ Poor oral hygiene was reported, as the cause of pregnancy gingivitis, more by the educated pregnant women than by less/ uneducated ones. This finding was analogous to the results showed by previous studies, wherein the authors have mentioned that the health behavior and utilization of health services are directly proportional to the level of education of pregnant women; the level of education significantly determined the good oral hygiene among pregnant women. ${ }^{18}$

In the present study, when the pregnant women were enquired about the measures they believed could prevent pregnancy gingivitis, majority of them (35.05\%) were aware of the fact that daily tooth brushing and flossing would be able to prevent the same. Respondents to the tune of $12.74 \%$ believed that getting dental checkup done during second trimester would prevent pregnancy gingivitis; and as many as $23.10 \%$ of the respondents believed that getting dental checkup done before pregnancy would be beneficial, while as $21.91 \%$ of them reported that balanced diet could prevent pregnancy gingivitis. According to the previous literature, all factors such as proper tooth brushing and flossing, ${ }^{10}$ regular dental checkups before and during pregnancy, and a balanced diet have their due role in preventing pregnancy gingivitis. In previous studies, it is reported that scaling, polishing, and root planning should be done irrespective of the stage/trimester of pregnancy so as to promote good oral health. ${ }^{19,20}$ It is found that less food intake during pregnancy (especially during first 
trimester) was associated with gingival bleeding due to deficient nutrients needed otherwise to protect the gingiva as well as the immune system from getting compromised. ${ }^{21}$

In the present study, around $55.37 \%$ of the respondents were unaware about the adverse pregnancy outcome of pregnancy gingivitis and $21.91 \%$ believed that it had no such adverse effect, while $13.54 \%$ of them reported preterm births as the adverse pregnancy outcome of pregnancy gingivitis. Many previous studies have found the correlation between periodontitis, preterm births, and low weight births. Also maternal periodontitis has been associated with poor oral health of child. ${ }^{22,23}$ Though the association of pregnancy gingivitis/periodontitis with adverse outcomes such as preterm births is still under research, it is better to maintain good oral health by pregnant women to avoid any associated complications as prevention has always been proven to be better than cure.

In the present study, most of the pregnant women (49.40\%) reported that using soft moist towel is the proper way of cleaning the oral cavity of children less than 18 months of age. It signifies that most of them were well versed with the way of oral hygiene maintenance among infants, though not all of them were aware about the same. Hence, educative programs for women should include the methods of oral hygiene measures and dental care of infants so as to prevent the development and progression of early childhood caries among infants. Importance of good oral hygiene among infants right from their birth should be emphasized upon so that they develop a healthy primary dentition that would result in the healthy permanent dentition to be carried along throughout life.

\section{Limitations}

1. The trimester of pregnancy was not taken into consideration.

2. The oral hygiene practices of the pregnant women were not explored nor were the participants examined for their gingival and periodontal status.

3. The stress level of subjects was not measured.

4. A convenience sample was taken that could limit generalizing of results on larger population.

\section{Conclusion}

Within the limits of the study, it was concluded that majority of the pregnant women were unaware about developing gingivitis during their pregnancy period. Most of the women did not know about various measures of prevention and treatment of pregnancy gingivitis and most of the pregnant women did not visit the dentist during pregnancy.

\section{Recommendations}

1. Oral health education program should include maternal oral health issues; women should be educated about the importance of good oral health especially during pregnancy and they should be made aware about its adverse effect on the oral health of new born.
2. Pregnant women should be counseled well for undergoing thorough dental examination before and during pregnancy that would prevent pregnancy gingivitis and its outcomes.

3. Preventive and education program should also include the topics regarding balanced, healthy diet needed for the well-being of the expecting mothers as well as the developing fetus.

4. It is recommended that pregnant women should undergo scaling/root planning at any stage of pregnancy without any fear.

5. It is recommended that the dentists should perform surgical procedures such as extractions, restorations, endodontic procedures in second or third trimester, after completion of fetal organogenesis. Moreover, dentists should be aware of the proper seating posture for pregnant women on a dental chair.

6. All health care professionals (dental/medical) should work in collaboration with each other so that they properly opt for relevant referrals for patients especially pregnant women.

\section{Conflict of Interest}

None declared.

\section{References}

1 Patil S, Thakur R, Madhu. K, Paul ST, Gadicherla P. Oral health coalition: knowledge, attitude, practice behaviours among gynaecologists and dental practitioners. J Int Oral Health 2013;5(1):8-15

2 Kandan PM, Menaga V, Kumar RR. Oral health in pregnancy (guidelines to gynaecologists, general physicians \& oral health care providers) J Pak Med Assoc 2011;61(10):1009-1014

3 Barak S, Oettinger-Barak O, Oettinger M, Machtei EE, Peled M, Ohel G. Common oral manifestations during pregnancy: a review. Obstet Gynecol Surv 2003;58(9):624-628

4 Tarsitano BF, Rollings RE. The pregnant dental patient: evaluation and management. Gen Dent 1993;41(3):226-234, quiz233-234

5 Lydon-Rochelle MT, Krakowiak P, Hujoel PP, Peters RM. Dental care use and self-reported dental problems in relation to pregnancy. Am J Public Health 2004;94(5):765-771

6 al-Kanhal MA, Bani IA. Food habits during pregnancy among Saudi women. Int J Vitam Nutr Res 1995;65(3):206-210

7 Michalowicz BS, DiAngelis AJ, Novak MJ, et al. Examining the safety of dental treatment in pregnant women. J Am Dent Assoc 2008;139(6):685-695

8 Xiong X, Buekens P, Fraser WD, Beck J, Offenbacher S. Periodontal disease and adverse pregnancy outcomes: a systematic review. BJOG 2006;113(2):135-143

9 Taani DQ, Habashneh R, Hammad MM, Batieha A. The periodontal status of pregnant women and its relationship with socio-demographic and clinical variables. J Oral Rehabil 2003;30(4):440-445

10 Bamanikar S, Kee LK. Knowledge, attitude and practice of oral and dental healthcare in pregnant women. Oman Med J 2013;28(4):288-291

11 Moawed S, Hawsawi A, Al Ahmed SS, Al-Atawi N, Awadien AZ. Knowledge and oral health care practices among Saudi pregnant women. Life Sci J 2014;11(5):32-41

12 Al-Swuailem AS, Al-Jamal FS, Helmi MF. Treatment perception and utilization of dental services during pregnancy among 
sampled women in Riyadh, Saudi Arabia. Saudi. J Dent Res 2014;5(2):123-129

13 George A, Johnson M, Blinkhorn A, Ellis S, Bhole S, Ajwani S. Promoting oral health during pregnancy: current evidence and implications for Australian midwives. J Clin Nurs 2010;19(23-24):3324-3333

14 Peruzzo DC, Benatti BB, Ambrosano GM, et al. A systematic review of stress and psychological factors as possible risk factors for periodontal disease. J Periodontol 2007;78(8):1491-1504

15 Kumar J, Samelson R, Oral Health Care during Pregnancy and Early Childhood: Practice Guidelines. New York, NY: New York State Department of Health; 2006

16 Lee A, McWilliams M, Janchar T. Care of the pregnant patient in the dental office. Dent Clin North Am 1999;43(3):485-494

17 Hey-Hadavi JH. Women's oral health issues: sex differences and clinical implications. Women's Health Prim Care 2002;5(3): 189-199
18 Yalcin F, Eskinazi E, Soydinc M, et al. The effect of sociocultural status on periodontal conditions in pregnancy. J Periodontol 2002;73(2):178-182

19 Trivedi S, Lal N, Singhal R. Periodontal diseases and pregnancy. J Orofac Sci 2015;7:67-68

20 Huda S, Doering H, Tenenbaum HC, Whittle W, Sigal MJ, Glogauer M. Oral neutrophil levels: a screening test for oral inflammatory load in pregnancy in a medical setting. J Periodontol 2015;86(1):72-81

21 Gaffar BO, El Tantawi MM, AlAgl AS, Al-Ansari A. Food intake, oral hygiene and gingival bleeding in pregnancy: does lifestyle make a difference? A cross sectional exploratory study. Int J Health Sci (Qassim) 2016;10(1):13-20

22 Casamassimo PS. Maternal oral health. Dent Clin North Am 2001;45(3):469-478,v-vi

23 Gajendra S, Kumar JV. Oral health and pregnancy: a review. N Y State Dent J 2004;70(1):40-44 


\section{Appendix A: Questionnaire}

Q1 Are you aware that women can develop pregnancy gingivitis (swollen red gums) during pregnancy?
(a) Yes, I am fully aware
(b) No, I am not aware
(c) I am not fully aware, but heard about it.

Q2 Did you get a dental checkup done before or during the period of your pregnancy?
(a) No, I did not
(b) Yes, I did
(c) I planned to, but did not.
(d) Yes, due to dental condition

Q3 Do you have any of the following signs of pregnancy gingivitis?
(a) Swollen and reddened gums
(b) Bad breath
(c) Gum bleeding
(d) Red spot over oral lining
(e) None

Q4 What according to your knowledge may be the cause of gingivitis (red and swollen gums) during pregnancy?
(a) Hormonal changes
(b) Traumatic tooth brushing
(c) Poor oral hygiene
(d) Eating fried food
(e) Malnutrition
(f) Do not know

Q5 What measures according to your knowledge can prevent pregnancy gingivitis?
(a) Daily tooth brushing and flossing
(b) Balanced diet
(c) Dental checkup before pregnancy
(d) Scaling every month
(e) Dental checkup during second trimester
(f) Do not know

Q6 What according to your knowledge is the treatment, dentists provide for pregnancy gingivitis?
(a) Surgical removal of swollen gum
(b) No treatment needed
(c) Professional scaling
(d) Extract the affected tooth
(e) Medication
(f) Do not know

Q7 What effect can pregnancy gingivitis have on your oral health?
(a) Overgrowth of gum tissue
(b) No effect
(c) Periodontal disease
(d) Tooth decay
(e) Tooth sensitivity
(f) Tooth becoming loose
(g) Do not know

Q8 Will pregnancy gingivitis have effect on oral health of your new born?
(a) Yes, it will
(b) No, it will not
(c) Undecided 
Q9 What effect can gingivitis possibly have on your pregnancy or the child to be born?
(a) Preterm birth
(b) No effect
(c) Malformation of bones
(d) Cleft lip and palate
(e) Do not know

Q10 What according to you is the proper way of cleaning the oral cavity of children less than 18 months of age?
(a) Tooth brush and paste
(b) Soft moist towel
(c) Electric tooth brush
(d) Mouthwash
(e) Saline

Q11 Do you suggest that pregnant women should have compulsory dental health education at the start of the pregnancy term?
(a) Yes
(b) No
(c) Undecided 